\title{
Mortality Risk Factors for Middle East Respiratory Syndrome Outbreak, South Korea, 2015
}

\section{Maimuna S. Majumder, Sheryl A. Kluberg, Sumiko R. Mekaru, John S. Brownstein}

As of July 15, 2015, the South Korean Ministry of Health and Welfare had reported 186 case-patients with Middle East respiratory syndrome in South Korea. For 159 casepatients with known outcomes and complete case histories, we found that older age and preexisting concurrent health conditions were risk factors for death.

$\mathrm{T}$ he ongoing outbreak of Middle East respiratory syndrome (MERS) in South Korea is the largest outside Saudi Arabia. As of July 15, the South Korean Ministry of Health and Welfare (MOHW) has reported 186 casepatients (1). Of these case-patients, 131 have recovered, 19 remained are hospitalized, and 36 had died (Figure 1). We conducted a preliminary mortality risk factor analysis for case-patients with MERS in South Korea who had known outcomes and covariates. We then compared our findings with those of previous investigations of case-patients in Saudi Arabia.

\section{The Study}

Case identification numbers were matched between the June 26, 2015, World Health Organization (WHO) line list (2) and daily text-based MERS reports from the South Korean MOHW (1). Matching between the 2 data sources was conducted by using age, sex, and date of reporting. The WHO line list included additional risk factor data, which were cross-validated against meta data from the MOHW. The MOHW daily MERS reports provided real-time outcome information.

As of July 15, outcomes and covariates were publicly available for 159 of 186 case-patients, all of whom became ill during weeks $2-7$ of the outbreak. We used this subset to describe the patient population, evaluate risk factors for death by using logistic regression models, and assess predictors of time from onset to diagnosis and onset to discharge by using Cox proportional hazards models.

Author affiliations: Massachusetts Institute of Technology, Cambridge, Massachusetts, USA (M.S. Majumder); Boston Children's Hospital, Boston, Massachusetts, USA (M.S. Majumder, S.A. Kluberg, S.R. Mekaru, J.S. Brownstein); Harvard Medical School, Boston (J.S. Brownstein)

DOI: http://dx.doi.org/10.3201/eid2111.151231
Five potential covariates were analyzed: sex, age, concurrent health condition status, health care worker status, and time from onset to diagnosis. For time-to-event analyses, patients were categorized into outbreak weeks by date of onset. We tested the Cox proportional hazards assumption by using Schoenfeld residuals and included an interaction term for predictor and follow-up time.

Of the 159 case-patients analyzed, 94 (59\%) were men. Per WHO definitions, 25 (16\%) had concurrent health conditions and $22(14 \%)$ were health care workers. Age was normally distributed (range 16-87 years, mean [SD] 55 [15.9] years). All deaths occurred in patients $>48$ years of age. Time from onset to diagnosis was positively skewed: median 4 days (interquartile range [IQR] 2-7 days). Median time from diagnosis to death and from diagnosis to discharge were 13 (IQR 17-25.3) and 22 (IQR 9-16.5) days, respectively.

As of July 15, a total of 35/159 cases analyzed were considered fatal, which yielded an estimated case-fatality rate (CFR) of $22 \%$. Univariate logistic regression models for each risk factor showed that older age and having a concurrent health condition were associated with death (both $\mathrm{p}<0.001$ ); both variables remained significant after we adjusted for all 5 variables in a multivariate logistic regression model (Table). The model estimated that odds of dying were 7 times higher for persons with concurrent health conditions than for persons without these conditions (odds ratio 7.14, 95\% CI 2.27-22.41). Furthermore, for every 1 -year increase in age, odds of dying increased by $12 \%$ (odds ratio 1.12, 95\% CI 1.07-1.17).

Time from onset to diagnosis decreased from a median of 10 days during outbreak week 2 (IQR 8.0-12.0 days) to 2 days during week 7 (IQR 1.0-2.0 days). There was a $43.7 \%$ average increase in hazard of diagnosis per week by a univariate Cox proportional hazards model $(\mathrm{p}<0.001)$. Separate univariate Cox models showed that no recorded risk factors were associated with this change.

Time from onset to discharge for patients who survived decreased from a median of 27 days during outbreak week 2 (IQR 22.0-32.0 days) to 19 days during week 7 (IQR 17.0-23.0). Univariate Cox proportional hazards analyses estimated a $34 \%$ average increase in the hazard of discharge per week ( $\mathrm{p}<0.001)$, a $63 \%$ increase for health care workers $(p=0.046)$, and an $8 \%$ decrease for every 1-day increase in time-to-diagnosis. Multivariate analysis controlling for all risk factors showed that the increase in 


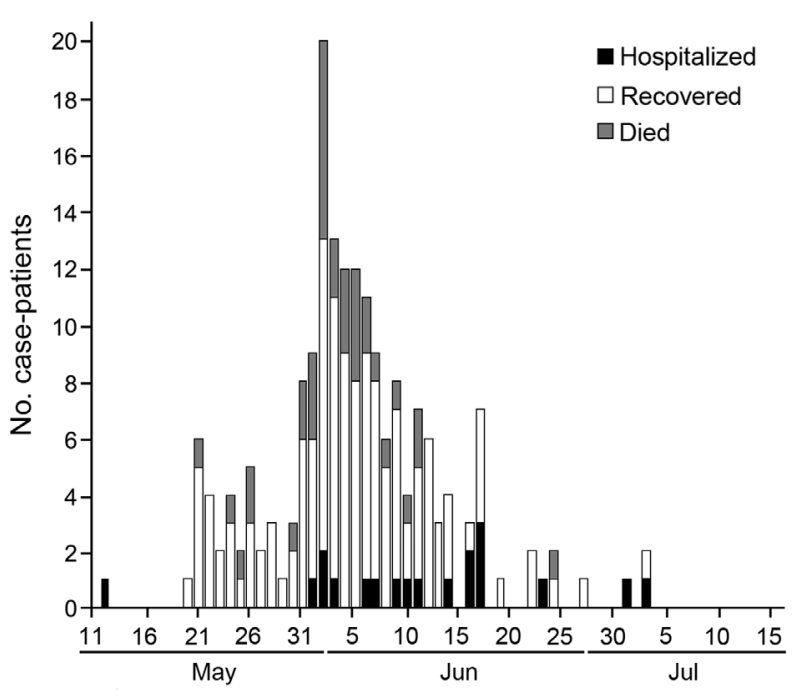

Date of symptom onset, 2015

Figure 1. New case-patients with Middle East respiratory syndrome, South Korea, by date of symptom onset and patient status, as of July 15,2015 . When date of symptom onset was unavailable, date of reporting was used. Although all 186 reported case-patients are included in this plot, only case-patients with known outcomes (e.g., recovered, died) and dates of onset were included in the analyses $(n=159)$.

hazard by week decreased to $26 \%(\mathrm{p}=0.032)$; no other covariates remained significant.

\section{Conclusions}

We found that older age and preexisting concurrent health conditions were associated with an increase in odds of death from MERS. Although being a health care worker appears to be protective, the association is not significant, probably because only 1 health care worker had died of MERS as of July 15, 2015. Time from onset to diagnosis was not an indicator for death, which suggests that the rapidity with which a patient receives supportive care may be of marginal consequence. Similarly, although being a male patient seems to increase odds of death, this relationship was not significant.

On the basis of case-patients who had known outcomes through July 15, the ongoing MERS outbreak in South Korea had an estimated CFR $(22 \%)$ that was half the CFR (44\%) for all known case-patients with MERS in
Saudi Arabia (3), but a CFR similar to that calculated for patients with only nonsporadic illness (21\%) (4). Because $19(10 \%)$ of 186 case-patients reported remain hospitalized, the final CFR of the outbreak might be higher than our current estimate. However, the proportion of patients who died (18\%-19\%) has been fairly stable since June 27 (Figure 2), which might indicate an asymptotic approach toward the final outbreak-specified CFR (5-7). If so, the final CFR associated with the MERS outbreak in South Korea during 2015 might be $<22 \%$. If all remaining hospitalized case-patients died, the final outbreak CFR would be $29 \%$, which provides an upper limit for our current estimate of $22 \%$ excluding additional cases.

A total of $16(64 \%)$ case-patients with MERS in South Korea who had concurrent health conditions died, compared with 19 (14\%) case-patients without concurrent health conditions. This finding is comparable to that in a MERS study in Saudi Arabia, which reported a 60\% CFR for a study population in which $45(96 \%)$ patients had concurrent health conditions (8). Although only $25(16 \%)$ case-patients had documented concurrent health conditions, the MERS outbreak in South Korean during 2015 has been largely nosocomial in nature. This finding suggests that observed differences between average CFRs in South Korea and Saudi Arabia might be driven in part by differential rates of concurrent health conditions for susceptible persons.

Use of publicly available data poses unique challenges. Although such data enable preliminary epidemiologic research during an ongoing outbreak, case information is stringently restricted to protect patient privacy. Because of this limitation, a follow-up analysis will be conducted pending availability of additional covariate data on potentially relevant biometrics (e.g., blood pressure) and behaviors (e.g., tobacco use), as well as outcomes for patients still hospitalized.

Despite these limitations, we found that risk factors for death among patients with MERS in South Korea who had known outcomes (age and concurrent health conditions) were similar to those identified for MERS case-patients in Saudi Arabia (8-10). Given these epidemiologic similarities and assuming that inherent virulence of MERS coronavirus is not context specific, the CFR difference might be caused not only by differential prevalence of risk factors but also by treatment or surveillance disparities.

\begin{tabular}{|c|c|c|c|}
\hline Variable & Value & Odds ratio $(95 \% \mathrm{Cl})$ & $p$ value \\
\hline Male sex, no. (\%) & $94(59)$ & $2.85(0.98-8.20)$ & 0.052 \\
\hline Mean (SD) age, y & $55(15.9)$ & $1.12(1.07-1.17)$ & $<0.001$ \\
\hline Concurrent health condition, no. (\%) & $25(16)$ & $7.14(2.27-22.41)$ & $<0.001$ \\
\hline Health care worker, no. (\%)† & $22(14)$ & $0.88(0.09-8.93)$ & 0.915 \\
\hline Median time-to-diagnosis, mo (IQR) & $4(2-7)$ & $1.00(0.89-1.14)$ & 0.957 \\
\hline
\end{tabular}




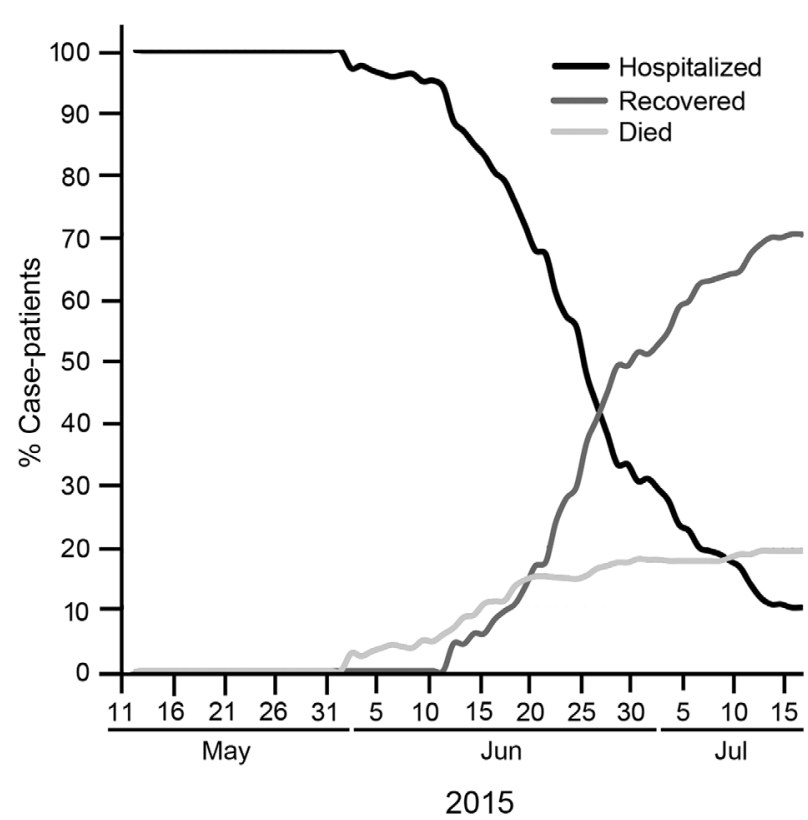

Figure 2. Cumulative proportion of case-patients with Middle East respiratory syndrome who were hospitalized, recovered, and died, South Korea, as of July 15, 2015. Total cumulative cases over time were calculated by date of symptom onset. When date of onset was unavailable, date of reporting was used. Cumulative recoveries and deaths over time were calculated by date of outcome; when date of outcome was unavailable, date of reporting was used. Although all 186 reported case-patients are included in this plot, only case-patients with known outcomes (e.g., recovered, died) and dates of onset were included in the analyses $(n=159)$.

Time to diagnosis decreased during the first 7 outbreak weeks, which probably contributed to the reduced length of hospitalization for patients who recovered and indicates that supportive care in South Korea might be highly adaptive. Furthermore, as reported by Cowling et al. (11), intensive case-finding activities might have produced more comprehensive diagnosis and reporting, thereby capturing less severe cases. In either event, given the frequency of importation events (12) and propensity for super-spreading (13), these findings provide information about MERS and MERS coronavirus in South Korea that might be useful in improving early case detection and preventing death.

This study was supported by the National Library of Medicine, National Institutes of Health (grant R01LM010812).

Ms. Majumder is an engineering systems doctoral student at the Massachusetts Institute of Technology, Cambridge, Massachusetts, and computational epidemiology research fellow at Boston Children's Hospital, Boston, Massachusetts.
Her research interests involve probabilistic modeling, Bayesian statistics, and systems epidemiology in the context of emerging infectious diseases.

\section{References}

1. South Korea Ministry of Health and Welfare. Press releases. July 21, 2015 [cited 2015 Jul 21]. http://www.mw.go.kr/front_new/ al/sal0301ls.jsp?PAR_MENU_ID $=04 \& M E N U \_I D=0403$

2. World Health Organization. MERS-CoV cases in the Republic of Korea as of 6/26/2015 [cited 2015 Jul 21]. http://www.who.int/entity/ emergencies/mers-cov/MERS-CoV-cases-kor-26Jun15.xlsx?ua=1

3. Saudi Arabia Ministry of Health. MOH: 1 new confirmed corona cases recorded, July 21, 2015 [cited 2015 Jul 21]. http://www.moh.gov.sa/en/CCC/PressReleases/Pages/ Statistics-2015-07-21-001.aspx

4. Cauchemez S, Fraser C, Van Kerkhove MD, Donnelly CA, Riley S, Rambaut A, et al. Middle East respiratory syndrome coronavirus: quantification of the extent of the epidemic, surveillance biases, and transmissibility. Lancet Infect Dis. 2014;14:50-6. http://dx.doi.org/10.1016/S1473-3099(13)70304-9

5. Nishiura H, Klinkenberg D, Roberts M, Heesterbeek JA. Early epidemiological assessment of the virulence of emerging infectious diseases: a case study of an influenza pandemic. PLoS ONE. 2009;4:e6852. http://dx.doi.org/10.1371/journal.pone.0006852

6. Galvani AP, Lei X, Jewell NP. Severe acute respiratory syndrome: temporal stability and geographic variation in case-fatality rates and doubling times. Emerg Infect Dis. 2003;9:991-4. http://dx.doi.org/10.3201/eid0908.030334

7. Ghani AC, Donnelly CA, Cox DR, Griffin JT, Fraser C, Lam TH, et al. Methods for estimating the case fatality ratio for a novel, emerging infectious disease. Am J Epidemiol. 2005;162:479-86. http://dx.doi.org/10.1093/aje/kwi230

8. Assiri A, Al-Tawfiq JA, Al-Rabeeah AA, Al-Rabiah FA, Al-Hajjar S, Al-Barrak A, et al. Epidemiological, demographic, and clinical characteristics of 47 cases of Middle East respiratory syndrome coronavirus disease from Saudi Arabia: a descriptive study. Lancet Infect Dis. 2013;13:752-61. http://dx.doi.org/ 10.1016/S1473-3099(13)70204-4

9. Saad M, Omrani AS, Baig K, Bahloul A, Elzein F, Matin MA, et al. Clinical aspects and outcomes of 70 patients with Middle East respiratory syndrome coronavirus infection: a single-center experience in Saudi Arabia. Int J Infect Dis. 2014;29:301-6. http://dx.doi.org/10.1016/j.ijid.2014.09.003

10. Assiri A, McGeer A, Perl TM, Price CS, Al Rabeeah AA, Cummings DA, et al. Hospital outbreak of Middle East respiratory syndrome coronavirus. N Engl J Med. 2013;369:407-16. http://dx.doi.org/10.1056/NEJMoa1306742

11. Cowling BJ, Park M, Fang VJ, Wu P, Leung GM, Wu JT. Preliminary epidemiological assessment of MERS-CoV outbreak in South Korea, May to June 2015. Euro Surveill. 2015;20:pii: 21163.

12. World Health Organization. Global map of countries with confirmed cases of MERS-CoV, July 8, 2015 [cited 2015 July 21]. http://www.who.int/csr/disease/coronavirus_infections/ mers-cov-global-situation-map-2015-07-08.png?ua=1

13. Kucharski AJ, Althaus CL. The role of superspreading in Middle East respiratory syndrome coronavirus (MERS-CoV) transmission. Euro Surveill. 2015;20:pii: 21167.

Address for correspondence: Maimuna S. Majumder, Boston Children's Hospital, 1 Autumn St, Boston, MA 02115, USA; email:maimuna@mit.edu 